\section{Radiopharmaceutical Chemistry}

\author{
J.S. Lewis, A.D. Windhorst, B.M. Zeglis, Eds.
}

Cham, Switzerland: Springer, 2019, eBook: $\$ 169.00$

Radiopharmaceutical chemistry is an essential part for promotion and development of nuclear medicine. This book is written by about 100 experts in radiopharmaceutical chemistry, with a direction and purpose not only as a collection of reviews but also as an educational opportunity, with many illustrations and tables. Thus, Radiopharmaceutical Chemistry might best be suited for postgraduate students and postdocs in the field of radiopharmaceutical chemistry. Senior class undergraduate students might also read this book. Nuclear medicine physicians who want to get a comprehensive view of the field and development trend of radiopharmaceuticals might be helped greatly as well. This book comprises 3 parts-Part I, First Principles; Part II, Radiochemistry; and Part III, Special Topics.

Part I contains 10 introductory and overview chapters. The first chapter starts with the introduction of nuclear imaging and radiotherapy. A short history of nuclear medicine is provided in the second chapter. The next 4 chapters include theorical basics about nuclear chemistry, production of radionuclides for nuclear medicine, and overviews of targeted therapy and nuclear imaging using radiopharmaceuticals. Fundamental concepts of targeting by small molecules, peptides, immunoglobulins, and nanoparticles as radiopharmaceutical vectors are introduced in the last 4 chapters of part I.

Part II comprises 14 chapters of radiopharmaceutical chemistries of various radionuclides from basics to some up-to-date information. The chapters are divided by radionuclides and synthetic or radiolabeling mechanisms. The first 2 chapters are about ${ }^{11} \mathrm{C}$ chemistry. Radiochemistries of ${ }^{13} \mathrm{~N}$ and ${ }^{15} \mathrm{O}$ are discussed in the third chapter. Interestingly, the fourth chapter is about the chemistry of gallium and indium radionuclides, though it might be expected that ${ }^{18} \mathrm{~F}$ chemistry information would be presented before this chapter as gallium and indium are metallic radionuclides and thus have properties more in common with the radionuclides discussed after the ${ }^{18} \mathrm{~F}$-related chapters. The development and use of ${ }^{68} \mathrm{Ga}$ is increasing rapidly due to the distribution of ${ }^{68} \mathrm{Ge} /{ }^{68} \mathrm{Ga}$ generators and the development of theranostics. Three ${ }^{18} \mathrm{~F}$ chemistry chapters based on the nucleophilic, electrophilic, and next-generation fluorination methods follow. The radiolabeling of ${ }^{18} \mathrm{~F}$ using aluminum complex in aqueous solution and various radiolabeling methods of arenes using ${ }^{18} \mathrm{~F}$-fluoride are introduced. ${ }^{99 \mathrm{~m}} \mathrm{Tc}$ radiopharmaceutical chemistries including the ${ }^{99} \mathrm{Mo} /{ }^{99 \mathrm{~m}} \mathrm{Tc}$ generator, current radiopharmaceuticals, and next-generation radiopharmaceuticals are discussed in the eighth chapter of part II; this chapter seems short compared with the importance of ${ }^{99 \mathrm{~m}} \mathrm{Tc}$ in nuclear medicine and radiopharmaceutical sciences. In-hospital procedures for radiolabeling of cells such as red blood cells or white blood cells and quality control for ${ }^{99 \mathrm{~m}} \mathrm{Tc}-$

COPYRIGHT @ 2019 by the Society of Nuclear Medicine and Molecular Imaging. labeled radiopharmaceuticals are not included. Radiopharmaceutical chemistry of copper radionuclides follows as a separate chapter. The tenth chapter in part II describes the important $\beta$-emitters ${ }^{90} \mathrm{Y}-$ and ${ }^{177} \mathrm{Lu}$ and radiopharmaceuticals labeled with these, which are hot issues recently due to theranostics. A positron emitter, ${ }^{89} \mathrm{Zr}$, described in the next chapter, might be a promising radionuclide for PET with long-circulating agents such as antibodies. Radioiodination mechanisms and preparation methods of various radioiodine-labeled radiopharmaceuticals are discussed in the twelfth chapter of part II. Production and radiolabeling methods of various $\alpha$-emitters follow in the next chapter. The last chapter of part II is radiopharmaceutical chemistry of seldom-used radionuclides such as ${ }^{38} \mathrm{~K},{ }^{82} \mathrm{Rb},{ }^{82 \mathrm{~m}} \mathrm{Rb},{ }^{82} \mathrm{Sr},{ }^{83} \mathrm{Sr},{ }^{89} \mathrm{Sr}$, and ${ }^{223} \mathrm{Ra}$.

Part III is about special topics. For example, bioconjugation methods and click chemistry are described in the first 2 chapters of part III. A chapter on equipment and instrumentation for radiopharmaceutical chemistry follows. Other topics besides chemistry but important for the practical radiopharmacy, such as kinetic modeling, radiation protection, and biostatistics, are covered in successive chapters. Important clinical points that should be considered when developing new radiopharmaceuticals for imaging or therapy are discussed in the seventh chapter of part III. Target identification and lead discovery requiring in vitro cell binding assay in the process of radiopharmaceutical development are included in the chapter after that. The next 2 chapters discuss preclinical experimentation in oncology and neurology. Clinical translation in Europe and in the United States are discussed in the eleventh and twelfth chapters of part III, and the final chapter is about setting up a successful radiopharmaceutical production facility including facility workflow design, validation of equipment and process, quality control methods, staffing and workflow, and the quality assurance system.

This book contains up-to-date radiopharmaceutical chemistry but not much cutting-edge knowledge, which can only be obtained in recent publications in this field. Thus, as mentioned earlier, this book can be adapted as an excellent textbook for postgraduate students or postdocs for radiopharmaceutical chemistry or molecular imaging. This book might be also helpful to experts in radiopharmaceutical chemistry, offering various useful information and allowing these experts to extend their knowledge in this field. Although there is a lack of clinical application of radiopharmaceuticals, this book might be helpful to obtain fundamental knowledge and inspirations about radiopharmaceuticals for nuclear medicine physicians. It's my great pleasure to adopt this new excellent book as the textbook for my next semester postgraduate lecture, and I greatly appreciate the editors and authors.

Jae Min Jeong

Seoul National University College of Medicine 28 Yungun-dong Jongro-gu Seoul, 110-744, Republic of Korea

E-mail:jmjng@snu.ac.kr

Published online Oct. 4, 2019.

DOI: 10.2967/jnumed.119.237479 